\title{
Tick-borne encephalitis virus in an Alsatian forest, eastern France: seasonal and annual variation of epidemiological parameters related to nymph-to-larva transmission and exposure of small mammals
}

\author{
Laure Bournez $^{1}$, Gérald Umhang ${ }^{1}$, Marie Moinet ${ }^{1}$, Céline RICHOMME ${ }^{1}$, Jean-Michel \\ Demerson $^{1}$, Christophe Caillot ${ }^{1}$, Elodie Devillers ${ }^{2}$, Jean-Marc Boucher ${ }^{1}$, Yves Hansmann ${ }^{3}$, \\ Franck Boue ${ }^{1}$, and Sara Moutailler ${ }^{2}$ \\ ${ }^{1}$ Anses \\ ${ }^{2}$ UMR BIPAR, Animal Health Laboratory \\ ${ }^{3}$ University Hospitals Strasbourg
}

April 28, 2020

\begin{abstract}
Tick-borne encephalitis virus (TBEV) is responsible for the most serious human viral tick-borne disease in Europe. A greater knowledge of the natural foci of TBEV and their fluctuating characteristics is essential to better assess the temporal variations of TBEV cycle's epidemiological risks. To describe the seasonal and inter-annual variations of the TBEV-cycle epidemiological parameters related to TBEV nymph-to-larva transmission, exposure of small mammals to TBEV and tick aggregation on small mammals, a longitudinal survey was conducted over a 3-year period to monitor a TBEV focus in a mountain forest in Alsace, eastern France. We trapped small mammals and collected questing ticks five times per year from 2012 to 2014 . TBEV prevalence in questing nymphs was lower in 2013 than in 2012 and 2014, probably because small mammals (Myodes glaerolus and Apodemus flavicollis) were more abundant in 2012, reducing tick aggregation and co-feeding transmission between ticks. This prevalence was higher in autumn than spring, which suggests that nymph-to-larva transmission of TBEV mainly occurs in spring. Despite these variations in prevalence, the density of infected questing nymphs was constant over time, leading to a constant risk for humans. The seroprevalence of small mammals was also constant over time although the proportion of small mammals harbouring ticks varied between years and seasons. Our results draw attention to the importance of considering the complex relationship between small mammal densities, tick aggregation on small mammals, density of infected questing nymphs and prevalence of infected nymphs in order to forecast the risk of TBEV for humans.
\end{abstract}

\section{Introduction}

Tick-borne encephalitis virus (TBEV) is responsible for the most serious human viral tick-borne disease in Europe. This flavivirus affects the human central nervous system causing meningitis or meningoencephalitis with long-term sequelae, the more severe forms progressing into a loss of consciousness, coma and even death (Bogovic \& Strle, Franc, 2015). In Western Europe, only the European subtype (TBEV-Eur), the least virulent subtype, is present (Süss, 2011). TBEV-Eur is mainly transmitted to a host by a tick bite and is maintained in nature by a cycle involving ticks - mainly Ixodes ricinus - and small mammals, especially those belonging to the genus Apodemus and Myodes (Süss, 2011). I. ricinus require three blood meals during their lifecycle. The larvae mainly feed on small mammals, while nymphs feed on medium-sized mammals, birds and reptiles, and adults on large animals such as ungulates (Mannelli, Bertolotti, Gern, \& Gray, 2012). Each stage of the life cycle takes from several months to around one year to develop to the next, so the entire 
life cycle is generally completed in 2 or 3 years, although this can vary from 2 to 6 years depending upon the geographical location (Gray, Kahl, Lane, Levin, \& Tsao, 2016; Perret, Rais, \& Gern, 2004).

The virus is transmitted transstadially from one life stage of the tick to the next after the moult (e.g. from larva to nymph), and on rare occasions can be transmitted vertically from a female tick to its eggs (Süss, 2011). Ticks can also become infected while feeding on a host during a viraemic phase. However, the duration of viraemia among small mammals and thus their infectivity to ticks are commonly considered short (2-9 days) (Chunikhin \& Kurenkov, 1979; Ernek, Kozuch, Lichard, Nosek, \& Albrecht, 1963; Heigl \& Von Zeipel, 1966; Kozuch et al., 1981). A recent experimental study in bank voles (Myodes glareolus ) suggests that viraemia might last up to 28 days, and therefore be longer than previously thought, but infectivity to ticks was not tested (Michelitsch et al., 2019). Therefore, co-feeding transmission between infected nymphs and uninfected larvae when they feed in spatiotemporal proximity to each other on the same host is thought to be the main transmission mode of TBEV-Eur and the most efficient way to maintain TBEV-Eur in a given area (Sarah E. Randolph, 2011). This transmission can even occur through immune hosts, although its efficiency is reduced (Milan Labuda \& Randolph, 1999).

The generational transfer of TBEV from infected nymphs to uninfected larvae via the host upon which they are feeding is the critical life history event that defines the epidemiology of TBEV (Voordouw, 2015). Therefore, the main key fitness component of TBEV is defined by the number of infected larvae produced via co-feeding transmission and, to a lesser extent, via systemic transmission. Thus, an essential prerequisite for TBEV persistence in a given area is the synchronous activity of larvae and nymphs (S. E. Randolph, Green, Peacey, \& Rogers, 2000). The substantial aggregation of ticks on a limited number of hosts promotes nymphto-larva transmission of TBEV (i.e. the number of infected larvae produced) and TBEV-cycle persistence. Ticks follow the "20/80 Rule" (Woolhouse et al., 1997) whereby 20\% of the reservoir hosts feed about $80 \%$ of the ticks (C. Burri, Bastic, Maeder, Patalas, \& Gern, 2011; Mannelli et al., 2012; Perkins, Cattadori, Tagliapietra, Rizzoli, \& Hudson, 2003; S. E. Randolph, Miklisová, Lysy, Rogers, \& Labuda, 1999; Rosà, Pugliese, Ghosh, Perkins, \& Rizzoli, 2007). The propensity of ticks to aggregate depends partly on the tick-host contact rate (activity of questing ticks, host and tick abundance, host community structure). For instance, it has been shown that small mammals are subject to greater tick infestation and higher individual loads of ticks when their population densities are low, given that each individual host has a higher probability of contact with ticks (Kiffner, Vor, Hagedorn, Niedrig, \& Rühe, 2011; Perez et al., 2017; Rosà et al., 2007). Therefore, the intensity of tick aggregation on a host is a useful parameter to study because the number of larvae co-feeding with infected nymphs impacts nymph-to-larva transmission.

The density of TBEV-infected nymphs that quest for vertebrate hosts in the environment (DIN), the prevalence of infected questing nymphs (NIP), and the proportion of small mammals harbouring TBEV-specific antibodies (seroprevalence) are three more relevant ecological and epidemiological parameters to monitor in order to understand the TBEV cycle. As small mammals rarely harbour adult ticks, DIN is an indicator of the instantaneous risk of exposure to TBEV for small mammals and for larvae via the host upon which they are feeding. NIP is a measurement of the proportion of larvae that became infected while feeding on a competent host in the preceding months or year (i.e. the nymph-to-larva transmission rate) and moulted into nymphs. In turn, the nymph-to-larva transmission depends on the intensity of tick aggregation on a few hosts and on the propensity of infected nymphs to feed on small mammals, which can be indirectly measured by the TBEV seroprevalence of small mammals. The relationship between DIN, NIP and the seroprevalence of small mammals and the parameters that influenced these parameters are presented in Figure 1.

The natural foci of TBEV are fluctuating ecosystems having characteristics that vary both intra- and interannually. A greater knowledge of these natural foci and their fluctuating characteristics are essential to better assess temporal variations of the epidemiological risks of TBEV-Eur. However, very few studies have addressed this issue (Perez-Eid, Hannoun, \& Rodhain, 1992; Rosà et al., 2019; Zöldi, Papp, Rigó, Farkas, \& Egyed, 2015). Both tick (larvae and nymphs) and small mammal densities show large inter-annual and intra-annual fluctuations. The activity of $I$. ricinus depends on humidity and temperature, and is therefore seasonal. Depending upon meteorological and climatic conditions and host availability, the abundance peak 
for questing larvae is either in late spring-early summer (in northern and central Europe, including our study area in eastern France) or in autumn (in western Europe) (Gray et al., 2016; Perez et al., 2017; S. E. Randolph et al., 2000). In western and central Europe, the peak for questing nymphs usually occurs in spring and early summer followed by comparatively low-level activity in mid-summer. In many areas, a second and minor abundance peak is observed in early autumn (Gray et al., 2016). This second peak has been partly attributed in Switzerland to the early emergence of larvae that have fed in spring (Perret et al., 2004). In temperate European forests, most small mammal species of the genus Apodemus and Myodes start breeding in spring and their population size reaches a peak in summer or autumn before decreasing during the winter (Crespin et al., 2002; Pucek, Jędrzejewski, Jędrzejewska, \& Pucek, 1993; Stenseth, Viljugrein, Jędrzejewski, Mysterud, \& Pucek, 2002). Therefore, TBEV nymph-to-larva transmission and exposure of small mammals to TBEV may vary seasonally as tick densities, TBEV-tick prevalence and their aggregation on host varies. Early spring, late spring/early summer and late summer/early autumn are seasons of particular interest for studying the TBEV epidemiological cycle.

In temperate forests, small mammal populations of the genusApodemus and Myodes are also subject to irregular multiannual oscillations, with a year of peak abundance occurring after a year of a heavy seed crop of oak and beech, and followed by a year of crashed abundance (Crespin et al., 2002; Jensen, 1982; Pucek et al., 1993; Stenseth et al., 2002). By the annual fluctuation in the number of larvae they feed, the temporal variation in the small mammal population may lead to an annual fluctuation in the density of questing nymphs, with a higher nymph density the year after a peak in rodent density (Brugger, Walter, ChitimiaDobler, Dobler, \& Rubel, 2018; Krawczyk et al., 2020; Ostfeld, Levi, Keesing, Oggenfuss, \& Canham, 2018). The effects of these variations on TBEV nymph-to-larva transmission and on the exposure of small mammals to TBEV are not straightforward, as the intensity of tick aggregation also varies annually (Perez et al., 2017; Rosà et al., 2007). Another factor that can influence the temporal variation of TBEV nymph-to-larva transmission is the variation over time of the community structure of small mammals (the relative density and the proportion of each small mammal species). Indeed, tick burden and the transmission-competence of the host - i.e. the ability of the host species to foster and amplify nymph-to-larva transmission through co-feeding or systemic (viraemic) transmission -, vary from one small mammal species to another, such as Apodemus flavicollis and Myodes glareolus (Dizij \& Kurtenbach, 1995; Kurtenbach et al., 1995; Milan Labuda \& Randolph, 1999; Pérez, Kneubühler, Rais, \& Gern, 2012).

France is located on the western border of the known distribution of TBEV, with about ten cases reported each year since its discovery in 1968. Most human clinical cases of TBE have been reported in Alsace, a region in the extreme East of France bordering Germany and Switzerland (Hansmann et al., 2006; Velay et al., 2019). Contrary to the endemic area of TBEV in these neighbouring countries, the incidence rate in Alsace is low, with a yearly incidence of $0.5 / 100,000$ inhabitants on average. The epidemiological characteristics of TBEV's natural foci, such as the intensity of virus circulation and nymph-to-larva transmission over time, has not been thoroughly investigated in Alsace. The only study of TBEV in ticks and small mammals in France was conducted from 1970 to 1974 in a closed peri-urban forest (Neuhof forest) near Strasbourg, an Alsatian city (Perez-Eid et al., 1992). We therefore conducted a longitudinal study of a TBEV focus in an Alsatian mountain forest over a 3-year period. The aims of the present study were to characterise the intensity of virus circulation and to describe both seasonal and inter-annual variations of the TBEV cycle's epidemiological parameters: (i) the density of TBEV-infected questing nymphs (DIN) and the prevalence of TBEV in questing nymphs (NIP) related to TBEV nymph-to-larva transmission, (ii) the TBEV seroprevalence of small mammals related to their exposure to TBEV; (iii) the prevalence of tick infestations of small mammals as a proxy for the intensity of aggregation on hosts, since these parameters are well correlated. The seasonal and inter-annual results were then discussed in relation to the variation over time of the density of ticks, the density of small mammals and the community structure of small mammals.

\section{Materials and Methods}

\section{Study area}

The study focused on a 4.0-hectare area at Murbach $\left(47^{\circ} 55^{\prime} 03 \mathrm{~N}, 07^{\circ} 08^{\prime} 46 \mathrm{E}\right.$; average altitude of $\left.630 \mathrm{~m}\right)$ in 
Guebwiller valley, Alsace, which is the region where most of the human cases of TBEV have been reported in the last two decades (Hansmann et al., 2006) and where the virus was isolated from questing ticks in 2010 (unpublished data). The site is covered by mixed forests classified as Asperulo-Fagetum beech forests, with a predominance of Fagus sylvatica beech andAbies alba European silver fir.

\section{Questing tick sampling}

Questing tick were counted and collected by dragging a $1-\mathrm{m}^{2}$ white blanket over the same area as the rodent trapping grid (see below). The trapping grid was divided into 16 smaller grids and the blanket was dragged over three different 10-metre-long transects within these 16 grids. In all, 48 different transects totalling a surface area of $480 \mathrm{~m}^{2}$ were investigated within the 4.0-hectare area. Ticks were collected during the first week of the month, once a month from April to October in 2011 and from May to October in 2012, 2013 and 2014, with the exception of July and August 2011, August 2013 and October 2014 when no ticks were sampled. At the laboratory, ticks were first identified at species level based on their morphology using appropriate keys and descriptions (Pérez-Eid, 2007). All questing nymphs and adults were washed in $70 \%$ ethanol, rinsed twice in distilled water, dried, and stored at - 80degC until tested for TBEV.

\section{Small mammal trapping and sampling}

Small mammals were trapped from 2012 to 2014 five times per year, in mid-April and in each first week of June, July, September and October. The trapping grid consisted of 196 live-traps (14 X 14 Uggland special nr. 3, Grahnab, Sweden) set at $15 \mathrm{~m}$ intervals, covering a total area of 3.2 ha. For each session, traps were set for three consecutive nights and baited with carrots and sunflower seeds. Trapped rodents were individually marked with a transponder (Veterissimo Mini RWI-I, Vethica, France). Blood samples were taken once per session through the retro-orbital sinus. Species, body mass, sex and tick presence were recorded and then the animals were released at the point of capture. We used the minimum value of weight observed during a second capture session to define the threshold value below which an individual was considered juvenile. Accordingly, individuals weighting less than $14 \mathrm{~g}$ for M. glareolus and $16 \mathrm{~g}$ for A. flavicollis were considered juveniles. From April 2012 to July 2013, all the ticks found on rodents were collected. In June and July 2012, given the high number of small rodents captured, although all the animals were subject to blood sampling and examined for tick presence, we only counted and collected the ticks of a number of randomly-chosen animals infested by ticks. All the ticks and blood samples were kept at $+4 \mathrm{degC}$ until brought back to the laboratory. There, the blood clot was gently detached from the bottom of the Eppendorf tube before being centrifuged at $5000 \mathrm{rpm}$ for $5 \mathrm{~min}$ and the serum obtained was stored at $-20 \mathrm{deg} \mathrm{C}$ until tested for the presence of TBEV antibodies. Larvae were identified at genus level and nymphs/adults at species level based on their morphology using appropriate keys and descriptions (Perez-Eid, 2007). All the ticks were washed in $70 \%$ ethanol, rinsed twice in distilled water, dried, and stored at -80degC until tested for TBEV

\section{Laboratory analysis}

TBEV antibody detection in rodents

Serum samples were screened for the presence of TBEV antibodies, using an in-house indirect immunofluorescence assay developed to capture specific IgG antibodies. ELISA plates were coated ( $2 \mu \mathrm{g} /$ well $)$ with a purified SNAP-TBEV EDIII recombinant protein or a non-relevant SNAP. The recombinant SNAP-TBEV EDIII protein was produced on S2 cell lines from the synthetic gene encoding the soluble ecto-domain (residues E293 to E399) from TBEV strain Kumlinge A52 cloned into a pMT/BiP/V5-HisA vector (Life Technologies, France) according to the manufacturer's recommendations (Beck et al., 2015). For the nonrelevant SNAP, we chose a production with a $\mathrm{pMT} / \mathrm{BiP} / \mathrm{V} 5$-HisA vector alone without an added coding gene. After overnight coating with antigen the plates were saturated with $1 \%$ BSA in PBS solution. Assays were performed in duplicate with 100 $\mu$ l of serum samples (diluted 1:500 in PBS), tested on SNAP-TBEV EDIII recombinant protein and on the SNAP production on the same plate. Following incubation for 1 hour at $37^{\circ} \mathrm{C}$ the plates were washed three times in PBS-Tween $0.05 \%$. The second antibody was a polyclonal goat anti-mouse conjugated with horseradish peroxidase (Dako), diluted to 1:500. After 1 hour at $37^{\circ} \mathrm{C}$ the wells were washed four times with $300 \mu \mathrm{l}$ of washing buffer. The bound peroxidase was revealed with $100 \mu \mathrm{l} 1.8 \mathrm{mM}$ 
of o-Phenylenediamine (Invitrogen) in PBS and $0.02 \% \mathrm{H}_{2} \mathrm{O}_{2}$ according to the manufacturer's instructions. After $40 \mathrm{~min}$ in the dark at room temperature, the enzyme reaction was stopped with $0.5 \mathrm{M} \mathrm{H}_{2} \mathrm{SO}_{4}$, and the optical density (OD) was measured at $492 \mathrm{~nm}$ on an automatic plate reader.

The positive controls used to validate the ELISA plate protocol consisted of serum from two groups of three Swiss mice that had previously been immunised twice with the recombinant SNAP-TBEV EDIII protein and used at a dilution of 1:5000. The negative controls were three Swiss mice that had not been immunised twice with the recombinant SNAP-TBEV EDIII protein. The samples were considered positive when the duplicate mean OD values read for SNAP-TBEV EDIII minus the mean OD values read for SNAP was higher than 0.100 OD.

TBEV detection in questing and fed ticks

Only ticks collected from 2012 to 2014 were analysed. For questing ticks, adults were analysed individually and nymphs were analysed in a pool of one to five ticks. For fed ticks (collected on small mammals), larvae/nymphs were pooled per stage, engorgement status (fed, unfed), animals and month of capture with one to ten ticks per pool, and adults were analysed individually. Ticks were homogenised using $2.8 \mathrm{~mm}$ stainless steel beads in a Precellys 24 lyser/homogeniser (Bertin, France) at $5500 \mathrm{rpm}$ for 20s. RNA was extracted using the Nucleospin RNA II kit (Macherey Nagel, Germany) according to the manufacturer's instructions. Purified RNA was eluted into $50 \mu \mathrm{l}$ RNase-free water and stored at $-80^{\circ} \mathrm{C}$ until use. RNA samples were screened for TBEV by real-time RT-PCR targeting a 3' non-coding region of the TBEV genome with specific primers and probes (Schwaiger \& Cassinotti, 2003). Real-time RT-PCR Taqman assays were performed in a final volume of $20 \mu \mathrm{l}$ using the LightCycler 480 RNA Master Hydrolysis Probes master mix (Roche Applied Science, Germany) according to the manufacturer's instructions using $2 \mu \mathrm{l}$ of RNA template. Positive and negative (water) controls were included in each run. Real-time RT-PCR thermal cycling conditions were as follows: $63^{\circ} \mathrm{C}$ for $3 \mathrm{~min}, 95^{\circ} \mathrm{C}$ for $30 \mathrm{~s}, 45$ cycles at $95^{\circ} \mathrm{C}$ for $10 \mathrm{~s}$, then $60^{\circ} \mathrm{C}$ for $30 \mathrm{~s}$, followed by cooling at $40^{\circ} \mathrm{C}$ for $10 \mathrm{~s}$. Conventional RT-PCR followed by nested PCR using primers targeting the non-structural protein gene NS5 (Puchhammer-Stöckl, Kunz, Mandl, \& Heinz, 1995) were used to confirm the presence of TBEV in positive samples. Amplicons were sequenced by Eurofins MWG Operon (Germany), and then assembled using BioEdit software (Ibis Biosciences, Carlsbad). The online BLAST tool (National Center for Biotechnology Information) was used to compare results with published sequences listed in the GenBank sequence databases.

\section{Descriptive and statistical analyses}

Definition of three seasons

We defined three seasons: season 1 for early spring (beginning of nymph activity and of small mammal reproduction), season 2 for the end of spring/early summer (peak of nymph activity and of small mammal reproduction) and season 3 for end of summer/early autumn (decrease of nymph and small mammal abundance levels). Given the small number of positives, we grouped data per season.

For small mammals, season 1 corresponded to the captures of April; season 2 grouped the captures of early June and July; and season 3 grouped the captures of early September and October. For each season, we listed each small mammal individuals captured along with its tick infestation status, TBEV seropositive status, and the number of captures (one or two). An individual was considered to be infested by ticks and TBEV seropositive in season 2 (respectively season 3) if it was found to be infested by at least one tick and TBEV seropositive in June or/and July (respectively in September or/and October). Similarly, an individual found to be infested by ticks or TBEV seropositive in at least one of the five trapping sessions of the year (from April to October) was considered positive in the year.

For questing ticks, season 1 corresponded to data for early May; season 2 grouped data for early June and July; and season 3 grouped data for early August, September and October.

Descriptive and statistical analyses of tick density and TBEV infection prevalence in ticks 
The monthly density of questing ticks was estimated for each tick stage by the mean density of questing ticks along each sampling transect, and was expressed as the mean number of ticks per $100 \mathrm{~m}^{2}$ with a $95 \%$ confidence interval $(95 \% \mathrm{CI})$. The density of questing ticks was estimated per season by the mean of the monthly density of questing ticks in the corresponding months, and per year by summing the tick density for each season (= annual cumulated DON). Tick questing density per year was compared using the observed yearly peak density and the annual cumulated DON with a Mann-Whitney $U$ test.

As TBEV infection prevalence in ticks is usually low, having been described as lower than $1 \%$ in Alsace (Bestehorn et al., 2018; Perez-Eid et al., 1992), prevalence in ticks was expressed as the minimum infection rate per 100 tested (MIR) based on the assumption that a single tick was positive within a positive pool. Exact 95\% CIs were calculated on the basis of binomial distribution. For the 2012-2014 period, MIR was calculated per season for each year. The inter-annual and inter-seasonnal MIR of questing ticks was compared using Fisher's exact test. If no TBEV was detected in a sample of ticks, we calculated the minimum prevalence of TBEV that could be detected in ticks with a probability of $95 \%$ given the sampling size used by applying the formula proposed by Cannon (Cannon, 2001).

We calculated the density of infected nymphs (DIN) per season by multiplying the seasonal MIR divided by 100 by the seasonal density of questing nymphs, and the DIN per year by multiplying the annual MIR divided by 100 with the annual cumulated DON. We used the lower and upper limits of the $95 \%$ CI for MIR per season and year to estimate the lower and upper bounds of the $95 \%$ CI for DIN.

Descriptive and statistical analyses of rodent density, tick infestation and TBEV seroprevalence in rodents

Rodent density was calculated per session and per species according to the standardised closed population Schnabel method that takes into account multiple marking occasions (Schnabel, 1938). Since the captures were carried out on three consecutive days per session, we considered that the population was closed for each session. Rodent density per season was calculated using the mean of rodent density estimated for the corresponding months.

The tick infestation prevalence for small mammals was defined as the number of small mammals carrying at least one tick divided by the number of small mammals inspected. We calculated tick infestation prevalence per year, season, species and age class (juveniles vs. adults). Exact 95\% CIs were calculated on the basis of binomial distribution. We described the infestation of small mammals by larvae and nymphs in season 2 (the period of highest nymph density) of 2012 and 2013 by calculating (1) the mean number of larvae and nymphs per individual infested by ticks, (2) the proportion of small mammals infested by ticks carrying nymphs only or nymphs and larvae, and (3) the proportion of larvae feeding on small mammals that were also carrying nymphs. The results for 2012 and 2013 were compared using a Mann-Whitney $U$ test.

The tick infestation status of small mammals was modelled using a logistic GLM (generalised linear model) with a binomial distribution and logit link, and a binary response variable (absence of ticks $=0$; presence of ticks $=1)$. The variables considered were the small mammal species, sex, season, year, and the interactions between year and season (to allow seasonal patterns to vary between years) and between season and species (to mimic seasonal variation of small mammal community structure and density). We included the number of captures within the season as an offset term. Some animals were captured in several different seasons and constituted temporal pseudoreplication of individual hosts. We therefore ran logistic GLMs for those individuals with the tick infestation status in season 2 (respectively in season 3) as the response variable, the infestation status in season 1 (respectively season 2), species, sex and year as the explanatory variables and the number of captures in season 2 (respectively season 3) as an offset term. As the infestation status of the previous season had no effect on the infestation status of the following season, we considered that these pseudo-replications would not bias the results of the model.

We calculated the TBEV seroprevalence of small mammals per year, season and species, and its exact $95 \%$ CI based on binomial distribution. The seropositive status of captured animals was modelled using a logistic GLM as a function of the small mammal species, season and year. As few individuals were seropositive (see Results), we limited the number of variables included in the model. 
Collinearity was checked in the models ensuring a variance inflation factor (VIF) $<10$ (James, Written, Hastie, \& Tibshirani, 2017). The backward elimination of explanatory variables was used to identify the most parsimonious model with the smallest Akaike information criteria (AIC).

Statistical computations were performed in R 3.5.0 (R Development Core team, 2018). For all statistical tests, a p-value $<0.05$ was considered statistically significant.

Ethical statement . The experimental protocol with small mammals complied with EU Directive 2010/63/EU and was submitted to and approved by the French Ministry of Research (APAFIS no. 2015120215112678). All efforts were made to minimize animal suffering. The species studied are not protected in France or included in the IUCN Red List of threatened species in France. The animal trapping took place with permission from the landowners.

\section{Results}

\section{Questing tick densities}

All the ticks collected were identified as Ixodes ricinusspecies. Overall, questing I. ricinus nymphs and adults were found on vegetation in all the sampled months with a unimodal pattern (Figure 2). The main period of activity for both nymphs and adults occurred between April/May and early July. Questing larvae were found from April to October and in higher numbers from May to early July, suggesting a synchronous activity with nymphs.

Questing nymph densities varied significantly between years (Mann-Whitney $U$ test, p-value $<0.001$ ). The annual cumulative DON was higher in 2011 and 2013 (respectively 500 and 575 nymphs $/ 100 \mathrm{~m}^{2}$ with a maximum density per month of 270 and 350 nymphs $/ 100 \mathrm{~m}^{2}$ ) than in 2012 and 2014 (respectively 120 and 240 nymphs $/ 100 \mathrm{~m}^{2}$ with a maximum density per month of 110 and 150 nymphs/100 $\mathrm{m}^{2}$ ) (Figures 2 and 3 ). The annual cumulative density of questing adults fluctuated in line with the annual cumulative DON, although the annual variations were lower than for the nymphs. It varied from $12-18$ adults $/ 100 \mathrm{~m}^{2}$ in 2012 and 2014 to $25-28$ adults $/ 100 \mathrm{~m}^{2}$ in 2011 and 2013 .

\section{TBEV infection prevalence in questing ticks and DIN}

A total of 4,054 questing Ixodes ricinus ticks (7,070 nymphs in 570 pools, 219 females and 199 males in 200 pools) were tested for TBEV (Supplementary Table 1). An overall MIR of $0.11 \%$ (95\% CI: $0.05-0.22$ ) of positive questing nymphs was observed. MIR in nymphs was significantly lower in 2013 (0.03\%, 95\% CI: 0 - 0.15) than in 2012 and 2014 (0.21\%, 95\% CI: 0.08 - 0.43, Fisher's exact test, p-value $=0.03)$ whereas there was no significant difference between 2012 and 2014 (Fisher's exact test, p-value $=0.7$, Figure 3). In 2012 and 2014, the MIR in nymphs was significantly lower in season 1 and $2(0.13 \%, 95 \%$ CI: $0.04-0.34)$ than in season $3(0.92 \%, 95 \%$ CI: $0.19-2.67$, Fisher's exact test, p-value $=0.02)$ whereas there was no significant difference between seasons 1 and 2 (Fisher's exact test, p-value $=1$, Figure 3). No questing adult was found to be infected. The minimum TBEV prevalence that could be detected in adult ticks with a probability of $95 \%$, given the overall sampling size from 2012 to 2014 was $0.7 \%$. This suggests that the overall prevalence in adults was less than $0.7 \%$.

The annual cumulated DIN appeared lower in 2013 than 2012 and 2014 (Figure 3) but the difference was not significant (2013: 0.14 infected nymphs per $100 \mathrm{~m}^{2}$, 95\% CI: $0-0.76 ; 2012: 0.42$ infected nymphs per $100 \mathrm{~m}^{2}, 95 \%$ CI: $0.10-1.23$; 2014: 0.30 infected nymphs per $100 \mathrm{~m}^{2}, 95 \%$ CI: $\left.0.08-0.74\right)$. In other words, around 43 to 136 infected nymphs quested a host each year within the study area as a whole. The DIN was not different per season (Figure 3), ranging from 0.09 [95\% CI: $0.02-0.25$ ] to 0.15 [95\% CI: $0.02-0.54$ ].

The eight sequences obtained from questing ticks (collected in 2012, 2013 and 2014) had 100\% identity with each other. One sequence was deposited in GenBank (accession number: MT109187) and showed 99\% homology with reference sequences from European subtype strains isolated in ticks (Germany, GenBank KX268728; Finland, GenBank MK801808), and human cerebellum (Finland, GenBank MG589937).

\section{Small mammal abundance and tick infestation prevalence}


Over the 15 capture sessions (totalling 8,820 trapping nights), 1,371 captures were recorded in total, corresponding to 906 different rodents captured per session over the 15 sessions (hereinafter called rodent-sessions) and 564 different individuals over the same 15 sessions: 276 Myodes glareolus, 287 Apodemus flavicollis , 1 Microtus agrestis. Overall, 342 rodents were captured during two or more trapping sessions. Data on each individual's weight and sex were completed for more than $90 \%$ of individuals for each trapping session, except in September and October 2012 when 33 individuals died in traps due to the predation of a stone marten.

The populations of bank vole (M. glareolus ) and yellow-necked mice (A. flavicollis ) peaked in 2012 (453 individuals trapped), dropped dramatically in 2013 (32 individuals) and was intermediate in 2014 (85 individuals) (Figure 4). Every year, the density of small mammals peaked during season 2 (June and July). Both species were similarly abundant in 2012 whereas in 2013 and 2014 -during the years of the lowest small mammal abundances-, A. flavicollis was captured slightly more often than M. glareolus (Figure 4). In 2012, bank voles were more abundant than yellow-necked mice in April and in September-October. Overall, 74 individuals were captured as juveniles, of which nine (12\%), 41 (55\%) and 19 (26\%) were respectively captured in April, June and July.

The proportion of rodents infested at least once by ticks significantly varied between years and seasons (Table 1). For all years, the prevalence of tick infestation on rodents was the highest in season 2 compared to the two other seasons $\left(\mathrm{Chi}^{2}, 2012\right.$, p-value $<0.001,2013$, p-value $=0.001,2014, \mathrm{p}$-value $\left.<0.001\right)$. The prevalence of tick infestation was lower in 2012 than 2013 and 2014 except in season 1. In season 2, this prevalence varied from $44 \%$ (95\% CI: 38.8 - 49.3) in 2012 to $94.2 \%$ (95\% CI: 85.8 - 98.4) in 2013 and $100 \%$ (95\% CI: $71.5-100)$ in 2014.

The best GLM for tick infestation prevalence on rodents included the small mammal species, season, year, the interaction between season and year and the interaction between season and small mammal species (Figure 5). Small mammals were significantly more likely to be infested by ticks in season 2 than in season 1 and 3. Yellow-necked mice were about twice as more likely to be infested than bank voles in season 2 of 2012 . In other seasons and years, there was no significant difference in the prevalence of tick infestation between species.

A total of 349 larvae of Ixodes sp ., 17 nymphs of $I$. ricinus and 4 females of $I$. trianguliceps were collected on 152 rodent-sessions in 2012 and 2013. During June and July, the mean number of larvae and nymphs per infested rodent-session (the intensity of tick infestation) was higher in 2013 (5.9 ticks +/- 4.8) than in 2012 (2.2 ticks +/- 2.2) (Mann-Whitney $U$ test, p-value $=0.01$, Table 2$)$ and was not significantly different between species (Mann-Whitney $U$ test, 2012: p-value $=0.64,2013: \mathrm{p}$-value $=0.45$, Table 2). The proportion of rodent-sessions infested by nymphs and co-infested by larvae and nymphs was higher in 2013 (6/12 and $5 / 12$ respectively) than in $2012(3 / 67$ and $2 / 67$ respectively, Table 2 , Fisher's exact test, p-value $<0.001)$. As a consequence, only $2.7 \%$ (4/127) of larvae fed simultaneously with nymphs in 2012 vs. $57.8 \%$ (37/64) in 2013.

\section{Detection of TBEV antibodies in small rodents}

We analysed 448 serum samples from $249 \mathrm{M}$. glareolus and 381 from 274 A. flavicollis . Overall, $4.2 \%$ [95\% CI: $2.6-6.3$ ] of individuals and $2.8 \%$ [95\% CI: $1.8-4.1$ ] of rodent-sessions were seropositive for TBEV. This proportion did not differ significantly between years (Fisher's exact test, p-value $=0.43$ ), varying from 1.3 \% [95\% CI: $0.0-7.1$ ] in 2014 ( $\mathrm{n}=76$ individuals) to $4.7 \%$ [95\% CI: $2.9-7.2$ ] in 2012 ( $\mathrm{n}=422$ individuals). Seroprevalence levels were similar between seasons in 2012 (3.2-5.2\%, Fisher's exact test, p-value $=0.53)$ (Table 1), with TBEV antibodies detected from April to October. In 2013 and 2014, TBEV antibodies were inconsistently detected throughout the year (Table 1).

Among the 22 seropositive rodents, three and one rodents were re-captured respectively one and two months after the detection of their seroconversion. TBEV antibodies were detected in two consecutive months for only one individual. Two juvenile yellow-necked mice (out of 61 juveniles of both species tested) with a body mass of $11 \mathrm{~g}$ and $15 \mathrm{~g}$ tested seropositive. 
None of the variables included in the logistic model — species, year and season - had a significant effect on the probability that a rodent would be seropositive.

\section{Detection of TBEV in feeding ticks}

All the ticks collected on rodents in 2012 and 2013 were tested for TBEV. In 2012, a MIR of 1.1\% [95\% CI : $0.2-3.1]$ was found on larvae $(n=283)$ and no nymphs $(n=9)$ nor females $(n=4)$ were found to be infected. No larvae were found positive in season 1 . The MIR in larvae did not differ significantly between seasons 2 and 3 (season 2: 0.7\% [95\% CI: 0.0 - 3.7], season 3: 4.2\% [95\% CI:0.5 - 14.2], Fisher's exact test, p-value=0.15). TBEV was detected in larvae feeding on three rodent-sessions $(2.2 \%$ of rodent-sessions infested by ticks, $\mathrm{n}=137)$ : one seronegative A. flavicollis captured in June $(1 / 67$ rodents infested by ticks during the June session) and two seronegative $M$. glareolus captured in September $(2 / 22$ rodents infested by ticks during the September session). These animals were not re-captured later. None of the feeding ticks collected in 2013 were found to be infected. Overall, 31 larvae and one nymph feeding on eight TBEV-seropositive rodents were negative for TBEV.

The three sequences obtained from feeding ticks (collected in 2012) had 100\% identity with each other. One sequence was deposited in GenBank (accession number: MT109186) and showed 100\% homology with sequences obtained from questing ticks during our study, and $99 \%$ homology with reference sequences from European subtype strains isolated in ticks (Germany, GenBank KX268728 ; Finland, GenBank MK801808), and human cerebellum (Finland, GenBank MG589937).

\section{Discussion}

Knowledge about the seasonal and inter-annual variation of TBEV nymph-to-larva transmission and small mammal exposure to TBEV is still sparse, especially in France where only one study was conducted prior to this one. Our study describes and monitors the parameters related to nymph-to-larvae transmission and small mammal exposure during a 3-year period in a forest in Alsace, eastern France.

\section{Low circulation of TBEV in the studied site}

TBEV was detected in nymph ticks from 2012 to 2014 with a very low prevalence varying annually from $0.03 \%$ [95\% CI: $0-0.15])$ to $0.24 \%$ [95\% CI : $0.07-0.62$ ]. Although adult ticks are generally more frequently infected than nymphs (Carpi, Bertolotti, Rosati, \& Rizzoli, 2009; Gaumann, Muhlemann, Strasser, \& Beuret, 2010; Perez-Eid et al., 1992; Rieille et al., 2014), our study did not detect the virus in adult ticks. This could nonetheless be explained by our small sample size. The method of collecting ticks by blanket dragging is more suitable for collecting nymph ticks than for adult ticks of I. ricinus . Considering the number of adults tested for TBEV, its prevalence in adult ticks was estimated to be lower than $0.7 \%$. These estimations fit within the lower range values found in other studies in Europe, ranging from $<0.1$ to $5 \%$ (Süss, 2011), and are similar to those observed by Perez-Eid et al. (Perez-Eid et al., 1992) in the Neuhof forest in Alsace between 1970 to 1974 with a maximum prevalence of $0.12 \%$ [95\% CI : $0.04-0.44$ ] in nymphs and $0.77 \%$ $[95 \%$ CI : $0.37-1.42]$ in adults.

In rodents, anti-TBEV antibodies were detected from 2012 to 2014 in $0 \%-5.2 \%$ of individuals per season. It is worth noting that TBEV antibodies can only be detected during a short period between the $5^{\text {th }}$ and the $13^{\text {th }}$ day up to the $42^{\text {nd }}$ day post-infection (Achazi et al., 2011; Milan Labuda et al., 1997). This is consistent with the observed disappearance of antibodies on seropositive animals captured a second time in our study and the finding of seronegative animals carrying infected larvae. These animals may have been recently infected and not yet seroconverted. Therefore, TBEV seroprevalence in small mammals is a good proxy for the occurrence of new infections in a month, although it is impossible to distinguish antibodies due to maternal transfer from those developed after a recent infection. In small mammals, maternal antibodies generally last from 6 to 10 weeks (Gomez-Chamorro et al., 2019; Grindstaff, Brodie, \& Ketterson, 2003; Kallio et al., 2006) but this duration is unknown for TBEV. We captured two juveniles aged 3-4 weeks according to their weight that were seropositive. Considering the time needed for seroconversion after an infection, these antibodies were probably maternal antibodies. Although serological tests and sampling design vary according 
to studies, the seroprevalence we observed in 2012-2015 is similar to that found by Perez-Eid et al. in Alsace (Perez-Eid et al., 1992) (2.4\%) and also fits within the lower values found in western and central Europe (prevalence ranging from 1.6\% to 23\% (Achazi et al., 2011; Bakhvalova, Potapova, Panov, \& Morozova, 2009; Caroline Burri et al., 2012; Cagnacci et al., 2012; Knap et al., 2012; Kozuch, Gresíková, Nosek, Lichard, \& Sekeyová, 1967; Tonteri et al., 2011)). In 2012 and 2013, we also observed a small proportion of rodents from which feeding ticks acquired the virus $(2 \%)$ associated with a low prevalence in feeding ticks $(1.6 \%)$.

Our results in rodents and questing ticks therefore suggest a very low circulation of TBEV in our site. This strengthens the hypothesis that the virus circulates at a very low level in the natural foci of the Alsace region, located at the western boundary of TBEV distribution. Randolph et al. (S. E. Randolph et al., 1999) theorised that the strength of TBEV enzootic cycles was influenced by the number of larvae co-feeding with nymphs on small mammals. Like the results of Perez-Eid et al. (Perez-Eid, 1990) in Alsace in the 1970s, we observed a very low number of co-feeding ticks in June-July during the period of highest tick activity, especially in 2012 . We only captured $4.5 \%$ of rodents infested by nymphs in 2012 when rodent abundance was high, whereas $17 \%$ to $33 \%$ of rodents were reported to be infested by nymphs in TBEV-infected sites in Switzerland and Slovakia (C. Burri et al., 2011; S. E. Randolph et al., 1999). In addition, the mean infestation of larvae per rodent-session infested by ticks (2.2) and per rodent-session infested by nymphs (2) in 2012 was also lower than that observed in other TBEV-infected sites (from 10 to 80 larvae per infested rodent and from 5 to 65 larvae per host infested by nymphs) (C. Burri et al., 2011; S. E. Randolph et al., 1999; Rosà et al., 2007). However, the apparent difference in the number of feeding ticks in our study compared to other field observations might be slightly lower considering that our method for counting feeding ticks is not directly comparable with the methods employed in other field studies and may have increased the underestimation of the number of feeding ticks.

\section{Seasonal and inter-annual variation of TBEV prevalence in ticks and rodents in 2012-2014}

Although the variations were low, we detected an annual and seasonal effect on NIP (the TBEV prevalence of questing nymphs) but neither on DIN nor rodent seroprevalence. Our study site in 2012-2014 was characterised by a high inter-annual and inter-seasonal variation in small mammal and questing nymph densities. Annually, the peak of activity for questing larvae and nymphs, of larvae feeding on small mammals, and of small mammal density occurred during the same season, i.e. in June-July. The density of bank voles and yellow-necked mice started to decrease from September. This decrease was greater for yellow-necked mice than for bank voles, only $13 \%$ of yellow-necked mice being captured in September-October. This seasonality is similar to field observations in TBEV-infected sites in neighbouring countries (C. Burri et al., 2011; S. E. Randolph et al., 1999; Rosà et al., 2007), but differs from findings in Brittany, western France, where the peak of small mammal densities and of feeding larvae were found to occur in autumn (Perez et al., 2017). Our observations on inter-annual variations comply with previous observations (Kiffner et al., 2011; Krawczyk et al., 2020; Ostfeld et al., 2018; Perez et al., 2017; Rosà et al., 2007): we found that the density of questing nymphs was higher the year following a year of high rodent density and that the intensity of tick aggregation on small mammals was higher when the density of small mammals was low. Indeed, the prevalence of infestation by both stages - larvae and nymphs - and the mean infestation was higher in 2013 (a low-density year for small mammals) than in 2012 (a high-density for small mammals).

NIP was higher in both 2012 and 2014 compared than 2013; this is contrary to the density of questing nymphs, for which we found opposite variations. However, DIN was near-constant over those years. Therefore, our results suggest that the proportion of infected larvae produced was higher in 2013 when more larvae and nymphs fed on the same very few hosts because there were fewer hosts available. This led to a higher proportion of infected questing nymphs the year after. This is consistent with the fact that the proportion of larvae feeding on rodents infested by nymphs was higher in 2013 than 2012. Therefore, the inter-annual difference in NIP can be explained by the variation in aggregation intensity of ticks on rodents along with rodent abundance. However, the overall number of fed larvae produced was probably lower in 2013, the year of a lower abundance of small mammals. Consequently, the DIN stayed constant over the years. Our study was only conducted over a 3 -year period and the results cannot be generalised. Further investigations over a longer 
period would be needed to better understand the relationship between DON, DIN, NIP and small mammal density for TBEV. Few studies have investigated these relationships for other tick-borne pathogens amplified by rodents. In North America, Ostfeld et al. (Ostfeld et al., 2018) studied for 19 years the relationship between the Ixodes scapularis tick, the Borrelia burgdorferi sl. bacteria and the white-footed mouse (Peromyscus leucopus ). They found no effect of white-footed mouse density on NIP Borrelia burgdorferi sl. whereas DIN

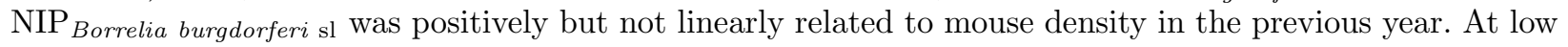
mouse density values, DIN Borreliaburgdorferi sl. was almost constant. In a short-term study in the Netherlands, Krawczyk et al. (Krawczyk et al., 2020) observed that the relationship between NIP, DIN and the rodent density the previous year depended on the transmission mode of the tick-borne pathogens. Contrary to most tick-borne pathogens amplified by rodents, TBEV is mainly transmitted by co-feeding and the nymph-tolarva transmission of TBEV strongly depends on the intensity of tick aggregation on small mammals. These elements probably induced a different relationship between DIN, NIP and rodent density that it would be interesting to investigate more.

Although the difference was small, TBEV prevalence in questing ticks was higher in autumn than in spring. This finding had already been observed in the previous study conducted in Alsace (Perez-Eid et al., 1992) and in studies targeting other tick-and-rodent-borne pathogens in Germany and Luxembourg (May, Jordan, Fingerle, \& Strube, 2015; Reye, Hubschen, Sausy, \& Muller, 2010). There are no data on the timings of diapause and moulting of I.ricinus stages in the Alsace region. However, if larvae that feed early in the season (in April-May) become nymphs that quest later the same year (in July-October) as observed in Switzerland (Perret et al., 2004), then this could be a potential mechanism to explain the higher prevalence in questing nymphs in autumn. Indeed, TBEV transmission to larvae might be higher in June-early July since this period coincides in our study area with the peak abundance of questing larvae and nymphs, of larvae feeding on small mammals and of small mammals themselves, especially Apodemus sp ., which has been shown experimentally to be more efficient in fostering TBEV transmission than $M$. glareolus (M. Labuda et al., 1993; Milan Labuda et al., 1997). Moreover, the virus titre may drop over time after the moult and in unfed ticks undergoing a winter diapause, as observed experimentally (Mishaeva and Erofeeva (Mishaeva \& Erofeeva, n.d.) cited by Perez-Eid et al. (Perez-Eid, 1990)), which would also induce lower prevalence in early spring.

Surprisingly, from 2012 to 2014 we did not detect any annual or seasonal effects on rodents' TBEV infection probability in keeping with the variation in the probability of a rodent being infested by ticks, whereas DIN values were constant. A higher seroprevalence could be expected in those years or seasons of low rodent abundance along with the higher prevalence of tick (nymph) infestation on rodents, as observed in other studies (Caroline Burri et al., 2012) and for other tick-borne pathogens amplified by rodents (Perez et al., 2017). Similarly, there was no species effect on the infection probability despite A. flavicollis being found to be more infested by ticks in this study (especially in spring when A. flavicollis is abundant). This apparent contrast may result from a difference in the immune response between species with a lower TBEV antibody titre and persistence in A. flavicollis compared with M. glareolus(Heigl \& Von Zeipel, 1966; Knap et al., 2012; Milan Labuda et al., 1996, 1997; Zeipel \& Heigl, 1966). We could then have expected to see an annual or seasonal difference in the infection probability per species, but our sample size of rodents was probably too small to detect a significant difference given the low seroprevalence of rodents, the low persistence of the antibodies and the low DIN. Another explanation for the absence of any annual effect on small mammal seroprevalence could be a lower detection of seropositive small mammals the years when they were scarce. Indeed, during those years, small mammals may acquire the infection much earlier in the year given the high tick aggregation level when fewer hosts are available. The exposure to this infection would probably remain undetected since the anti-TBEV antibodies' half-life is very short in small mammals.

In conclusion, this study shows that the virus was circulating at a very low level in our study site. Despite this very low-level circulation, we were able to observe a significant variation in the inter-annual and interseasonal prevalence of TBEV in questing nymphs, indicating that the nymph-to-larva transmission of TBEV varied over time. However, the density of infected questing nymphs showed no detectable variations over time, suggesting that the rate of exposure for humans was probably relatively constant over the period of the study. 
The seroprevalence of small mammals was constant over time although the prevalence of tick infestation varied on an annual and seasonal basis. More studies are needed to understand the relationship between the density of TBEV-infected questing infected nymphs, the density of questing nymphs, the prevalence of TBEV in questing nymphs, TBEV seroprevalence in small mammals and small mammal density.

\section{Acknowledgements}

The authors are very grateful to all the volunteers who helped in the capture of small mammals and to the French forestry agency, ONF, which authorised trapping in Murbach forest. The authors would also like to thank Philippe Desprès, Institut Pasteur, Paris, France for providing recombinant proteins SNAP-TBEV EDIII and SNAP. We thank the "Tiques et Maladies a Tiques (TMT)" group "of the "Reseau Ecologie des Interactions Durables" (REID) for stimulating discussion and support.

\section{Funding}

This project was funded by ANSES through a transversal project grant called "PREV-TBE-TIQRO" from 2010 to 2012.

\section{Conflicts of interest}

The authors declare that they have no conflicts of interest.

\section{Data availability statement}

The data that support the findings of this study will be available online on a repository in zenodo after acceptance.

\section{References}

Achazi, K., Růžek, D., Donoso-Mantke, O., Schlegel, M., Ali, H. S., Wenk, M., ... Niedrig, M. (2011). Rodents as Sentinels for the Prevalence of Tick-Borne Encephalitis Virus. Vector-Borne and Zoonotic Diseases , 11 (6), 641-647. https://doi.org/10.1089/vbz.2010.0236

Bakhvalova, V. N., Potapova, O. F., Panov, V. V., \& Morozova, O. V. (2009). Vertical transmission of tick-borne encephalitis virus between generations of adapted reservoir small rodents. Virus Research ,140 (1-2), 172-178. https://doi.org/10.1016/j.virusres.2008.12.001

Beck, C., Desprès, P., Paulous, S., Vanhomwegen, J., Lowenski, S., Nowotny, N., .. Lecollinet, S. (2015). A high-performance multiplex immunoassay for serodiagnosis of flavivirus-associated neurological diseases in horses. BioMed Research International ,2015 , 1-13. https://doi.org/10.1155/2015/678084

Bestehorn, M., Weigold, S., Kern, W. V., Chitimia-Dobler, L., Mackenstedt, U., Dobler, G., \& Borde, J. P. (2018). Phylogenetics of tick-borne encephalitis virus in endemic foci in the upper Rhine region in France and Germany. PLOS ONE , 13 (10), e0204790. https://doi.org/10.1371/journal.pone.0204790

Bogovic, P., \& Strle, Franc. (2015). Tick-borne encephalitis: A review of epidemiology, clinical characteristics, and management. World Journal of Clinical Cases , 3 (5), 430-441. https://doi.org/10.12998/wjcc.v3.i5.430

Brugger, K., Walter, M., Chitimia-Dobler, L., Dobler, G., \& Rubel, F. (2018). Forecasting next season's Ixodes ricinus nymphal density: the example of southern Germany 2018. Experimental and Applied Acarology , 75 (3), 281-288. https://doi.org/10.1007/s10493-018-0267-6

Burri, C., Bastic, V., Maeder, G., Patalas, E., \& Gern, L. (2011). Microclimate and the zoonotic cycle of tick-borne encephalitis virus in Switzerland. Journal of Medical Entomology , 48 (3), 615-627. https://doi.org/10.1603/ME10180

Burri, C., Korva, M., Bastic, V., Knap, N., Avšič-Županc, T., \& Gern, L. (2012). Serological evidence of tick-borne encephalitis virus infection in rodents captured at four sites in Switzerland.Journal of Medical Entomology , 49 (2), 436-439. https://doi.org/10.1603/ME11084 
Cagnacci, F., Bolzoni, L., Rosà, R., Carpi, G., Hauffe, H. C., Valent, M., .. Rizzoli, A. (2012). Effects of deer density on tick infestation of rodents and the hazard of tick-borne encephalitis. I: Empirical assessment. International Journal for Parasitology ,42 (4), 365-372. https://doi.org/10.1016/j.ijpara.2012.02.012

Cannon, R. M. (2001). Sense and sensitivity - designing surveys based on an imperfect test. Preventive Veterinary Medicine ,49 (3-4), 141-163. https://doi.org/10.1016/S0167-5877(01)00184-2

Carpi, G., Bertolotti, L., Rosati, S., \& Rizzoli, A. (2009). Prevalence and genetic variability of tick-borne encephalitis virus in host-seeking Ixodes ricinus in northern Italy. Journal of General Virology ,90 (12), 2877-2883. https://doi.org/10.1099/vir.0.013367-0

Chunikhin, S. P., \& Kurenkov, V. B. (1979). Viraemia in Clethrionomys glareolus -a new ecological marker of tick-borne encephalitis virus.Acta Virologica , 23 (3), 257-260.

Crespin, L., Verhagen, R., Stenseth, N. C., Yoccoz, N. G., Prevot-Julliard, A.-C., \& Lebreton, J.-D. (2002). Survival in fluctuating bank vole populations: seasonal and yearly variations. Oikos , 98 (3), 467-479. https://doi.org/10.1034/j.1600-0706.2002.980311.x

Dizij, A., \& Kurtenbach, K. (1995). Clethrionomys glareolus, but not Apodemus flavicollis, acquires resistance to lxodes ricinus L, the main European vector of Borrelia burgdorferi. Parasite Immunology ,17 (4), 177-183. https://doi.org/10.1111/j.1365-3024.1995.tb00887.x

Ecker, M., Allison, S. L., Meixner, T., \& Heinz, F. X. (1999). Sequence analysis and genetic classification of tick-borne encephalitis viruses from Europe and Asia. Journal of General Virology , 80 (1), 179-185. https://doi.org/10.1099/0022-1317-80-1-179

Ernek, E., Kozuch, O., Lichard, M., Nosek, J., \& Albrecht, P. (1963). Experimental Infection of Clethrionomys glareolus and Apodemus flavicollis with Tick-Borne Encephalitis Virus. Acta Virologica ,7 (5), 434-436.

Gaumann, R., Muhlemann, K., Strasser, M., \& Beuret, C. M. (2010). High-throughput procedure for tick surveys of tick-borne encephalitis virus and its application in a national surveillance study in Switzerland. Applied and Environmental Microbiology ,76 (13), 4241-4249. https://doi.org/10.1128/AEM.00391-10

Gomez-Chamorro, A., Heinrich, V., Sarr, A., Roethlisberger, O., Genné, D., Bregnard, C., .. Voordouw, M. J. (2019). Maternal antibodies provide bank voles with strain-specific protection against infection by the Lyme disease pathogen. Applied and Environmental Microbiology , 85 (23). https://doi.org/10.1128/AEM.0188719

Gray, J. S., Kahl, O., Lane, R. S., Levin, M. L., \& Tsao, J. I. (2016). Diapause in ticks of the medically important Ixodes ricinus species complex. Ticks and Tick-Borne Diseases , 7 (5), 992-1003. https://doi.org/10.1016/j.ttbdis.2016.05.006

Grindstaff, J. L., Brodie, E. D., \& Ketterson, E. D. (2003). Immune function across generations: integrating mechanism and evolutionary process in maternal antibody transmission. Proceedings of the Royal Society of London. Series B: Biological Sciences ,270 (1531), 2309-2319. https://doi.org/10.1098/rspb.2003.2485

Hansmann, Y., Pierre Gut, J., Remy, V., Martinot, M., Allard Witz, M., \& Christmann, D. (2006). Tickborne encephalitis in eastern France.Scandinavian Journal of Infectious Diseases , 38 (6-7), 520-526. https://doi.org/10.1080/00365540600585073

Heigl, Z., \& Von Zeipel, G. (1966). Experimental infection with tick-borne encephalitis virus in Clethrionomys glareolus, Apodemus flavicollis, Apodemus sylvaticus and Mus musculus. 1. Virological Studies. Acta Pathologica Microbiologica Scandinavica ,66 (4), 489-509. https://doi.org/10.1111/apm.1966.66.4.489

James, G., Written, D., Hastie, T., \& Tibshirani, R. (2017). An Introduction to Statistical Learning (8th ed.). (Springer Science+Business Media New York).

Jensen, T. S. (1982). Seed production and outbreaks of non-cyclic rodent populations in deciduous forests. Oecologia , 54 (2), 184-192. https://doi.org/10.1007/BF00378391 
Kallio, E. R., Poikonen, A., Vaheri, A., Vapalahti, O., Henttonen, H., Koskela, E., \& Mappes, T. (2006). Maternal antibodies postpone hantavirus infection and enhance individual breeding success.Proceedings of the Royal Society B: Biological Sciences ,273 (1602), 2771-2776. https://doi.org/10.1098/rspb.2006.3645

Kiffner, C., Vor, T., Hagedorn, P., Niedrig, M., \& Rühe, F. (2011). Factors affecting patterns of tick parasitism on forest rodents in tick-borne encephalitis risk areas, Germany. Parasitology Research , 108 (2), 323-335. https://doi.org/10.1007/s00436-010-2065-x

Knap, N., Korva, M., Dolinšek, V., Sekirnik, M., Trilar, T., \& Avšič-Županc, T. (2012). Patterns of tickborne encephalitis virus infection in rodents in Slovenia. Vector-Borne and Zoonotic Diseases , 12 (3), 236-242. https://doi.org/10.1089/vbz.2011.0728

Kozuch, O., Chunikhin, S., Gresikova, M., Nosek, J., Kurenkov, V., \& Lysý, J. (1981). Experimental characteristics of viraemia caused by two strains of tick-borne encephalitis virus in small rodents. Acta Virologica , 25 (4), 219-224.

Kozuch, O., Gresíková, M., Nosek, J., Lichard, M., \& Sekeyová, M. (1967). The role of small rodents and hedgehogs in a natural focus of tick-borne encephalitis. Bulletin of the World Health Organization, 36 Suppl , 61-66.

Krawczyk, A. I., van Duijvendijk, G. L. A., Swart, A., Heylen, D., Jaarsma, R. I., Jacobs, F. H. H., ... Takken, W. (2020). Effect of rodent density on tick and tick-borne pathogen populations: consequences for infectious disease risk. Parasites $\&$ Vectors , 13 (1). https://doi.org/10.1186/s13071-020-3902-0

Kurtenbach, K., Kampen, H., Dizij, A., Arndt, S., Seitz, H. M., Schaible, U. E., \& Simon, M. M. (1995). Infestation of Rodents with Larval Ixodes ricinus (Acari; Ixodidae) Is an Important Factor in the Transmission Cycle of Borrelia burgdorferi s.l. in German Woodlands. Journal of Medical Entomology , 32 (6), 807-817. https://doi.org/10.1093/jmedent/32.6.807

Labuda, M., Austyn, J. M., Zuffova, E., Kozuch, O., Fuchsberger, N., Lysy, J., \& Nuttall, P. A. (1996). Importance of localized skin infection in tick-borne encephalitis virus transmission. Virology , 219 (2), 357366. https://doi.org/10.1006/viro.1996.0261

Labuda, M., Kozuch, O., Zuffová, E., Elecková, E., Hails, R. S., \& Nuttall, P. A. (1997). Tick-borne encephalitis virus transmission between ticks cofeeding on specific immune natural rodent hosts. Virology , 235 (1), 138-143. https://doi.org/10.1006/viro.1997.8622

Labuda, M., Nuttall, P. A., Kozuch, O., Elecková, E., Williams, T., Zuffová, E., \& Sabó, A. (1993). Nonviraemic transmission of tick-borne encephalitis virus: a mechanism for arbovirus survival in nature. Experientia , $49(9), 802-805$.

Labuda, M., \& Randolph, S. E. (1999). Survival strategy of tick-borne encephalitis virus: Cellular basis and environmental determinants.Zentralblatt Für Bakteriologie , 289 (5-7), 513-524. https://doi.org/10.1016/S09348840(99)80005-X

Mannelli, A., Bertolotti, L., Gern, L., \& Gray, J. (2012). Ecology of Borrelia burgdorferi sensu lato in Europe: transmission dynamics in multi-host systems, influence of molecular processes and effects of climate change. FEMS Microbiology Reviews, 36 (4), 837-861. https://doi.org/10.1111/j.1574-6976.2011.00312.x

May, K., Jordan, D., Fingerle, V., \& Strube, C. (2015). <Borrelia burgdorferi sensu lato and co-infections with Anaplasma phagocytophilum and Rickettsia spp. in Ixodes ricinus in Hamburg, Germany: Borrelia infections in ticks in northern Germany. Medical and Veterinary Entomology , 29 (4), 425-429. https://doi.org/10.1111/mve.12125

Michelitsch, Tews, Klaus, Bestehorn-Willmann, Dobler, Beer, \& Wernike. (2019). In vivo characterization of tick-borne encephalitis virus in bank voles (Myodes glareolus). Viruses , 11 (11), 1069. https://doi.org/10.3390/v11111069 
Mishaeva, N. P., \& Erofeeva, N. I. (n.d.). The effect of diapause of the tick Ixodes ricinus (Ixodidae) upon the multiplication of the tick encephalitis virus in them. Parazitologiya , 13 (3), 218-222.

Ostfeld, R. S., Levi, T., Keesing, F., Oggenfuss, K., \& Canham, C. D. (2018). Tick-borne disease risk in a forest food web. Ecology ,99 (7), 1562-1573. https://doi.org/10.1002/ecy.2386

Pérez, D., Kneubühler, Y., Rais, O., \& Gern, L. (2012). Seasonality of Ixodes ricinus Ticks on Vegetation and on Rodents and Borrelia burgdorferi sensu lato Genospecies Diversity in Two Lyme Borreliosis-Endemic Areas in Switzerland. Vector-Borne and Zoonotic Diseases , 12 (8), 633-644. https://doi.org/10.1089/vbz.2011.0763

Perez, G., Bastian, S., Chastagner, A., Agoulon, A., Plantard, O., Vourc'h, G., \& Butet, A. (2017). Ecological factors influencing small mammal infection by Anaplasma phagocytophilum and Borrelia burgdorferi s.l. in agricultural and forest landscapes: Tick-borne infection in small mammals. Environmental Microbiology , 19 (10), 4205-4219. https://doi.org/10.1111/1462-2920.13885

Perez-Eid, C. (1990). Les relations tiques - petits mammifères dans le foyer Alsacien d'encéphalite à tiques. Acarologia , 31 (1), 131-141.

Pérez-Eid, C. (2007). Les tiques: Identification, biologie, importance medicale et veterinaire. Paris: TEC \& DOC Lavoisier.

Perez-Eid, C., Hannoun, C., \& Rodhain, F. (1992). The Alsatian tick-borne encephalitis focus: Presence of the virus among ticks and small mammals. European Journal of Epidemiology , 8 (2). https://doi.org/10.1007/BF00144797

Perkins, S. E., Cattadori, I. M., Tagliapietra, V., Rizzoli, A. P., \& Hudson, P. J. (2003). Empirical evidence for key hosts in persistence of a tick-borne disease. International Journal for Parasitology ,33 (9), 909-917. https://doi.org/10.1016/S0020-7519(03)00128-0

Perret, J.-L., Rais, O., \& Gern, L. (2004). Influence of climate on the proportion of Ixodes ricinus nymphs and adults questing in a tick population. Journal of Medical Entomology , 41 (3), 361-365. https://doi.org/10.1603/00222585-41.3.361

Pucek, Z., Jedrzejewski, W., Jedrzejewska, B., \& Pucek, M. (1993). Rodent population dynamics in a primeval deciduous forest (Białowieża National Park) in relation to weather, seed crop, and predation. Acta Theriologica , 38 , 199-232. https://doi.org/10.4098/AT.arch.93-18

Puchhammer-Stöckl, E., Kunz, C., Mandl, C. W., \& Heinz, F. X. (1995). Identification of tick-borne encephalitis virus ribonucleic acid in tick suspensions and in clinical specimens by a reverse transcription-nested polymerase chain reaction assay. Clinical and Diagnostic Virology , 4 (4), 321-326. https://doi.org/10.1016/09280197(95)00022-4

R Development Core team. (2018). R: A Language and Environment for Statistical Computing. http://www.Rproject.org/. Vienna, Austria R Foundation for Statistical Computing;

Randolph, S. E. (2011). Transmission of tick-borne pathogens between co-feeding ticks: Milan Labuda's enduring paradigm. Ticks and Tick-Borne Diseases , 2 (4), 179-182. https://doi.org/10.1016/j.ttbdis.2011.07.004

Randolph, S. E., Green, R. M., Peacey, M. F., \& Rogers, D. J. (2000). Seasonal synchrony: the key to tick-borne encephalitis foci identified by satellite data. Parasitology, 121 ( Pt 1) , 15-23.

Randolph, S. E., Miklisová, D., Lysy, J., Rogers, D. J., \& Labuda, M. (1999). Incidence from coincidence: patterns of tick infestations on rodents facilitate transmission of tick-borne encephalitis virus.Parasitology , 118 (2), 177-186. https://doi.org/10.1017/S0031182098003643

Reye, A. L., Hubschen, J. M., Sausy, A., \& Muller, C. P. (2010). Prevalence and seasonality of tick-borne pathogens in questing Ixodes ricinus ticks from Luxembourg. Applied and Environmental Microbiology , 76 (9), 2923-2931. https://doi.org/10.1128/AEM.03061-09 
Rieille, N., Bressanelli, S., Freire, C. C. M., Arcioni, S., Gern, L., Péter, O., \& Voordouw, M. J. (2014). Prevalence and phylogenetic analysis of tick-borne encephalitis virus (TBEV) in field-collected ticks (Ixodes ricinus) in southern Switzerland. Parasites 83 Vectors , 7 (1), 443. https://doi.org/10.1186/1756-3305-7-443

Rosà, R., Pugliese, A., Ghosh, M., Perkins, S. E., \& Rizzoli, A. (2007). Temporal variation of Ixodes ricinus intensity on the rodent host Apodemus flavicollis in relation to local climate and host dynamics. Vector-Borne and Zoonotic Diseases , 7 (3), 285-295. https://doi.org/10.1089/vbz.2006.0607

Rosà, R., Tagliapietra, V., Manica, M., Arnoldi, D., Hauffe, H. C., Rossi, C., .. Rizzoli, A. (2019). Changes in host densities and co-feeding pattern efficiently predict tick-borne encephalitis hazard in an endemic focus in northern Italy. International Journal for Parasitology , 49 (10), 779-787. https://doi.org/10.1016/j.ijpara.2019.05.006

Schnabel, Z. E. (1938). The estimation of total fish populations of a lake. The American Mathematical Monthly , 45 (6), 348-352.

Schwaiger, M., \& Cassinotti, P. (2003). Development of a quantitative real-time RT-PCR assay with internal control for the laboratory detection of tick borne encephalitis virus (TBEV) RNA. Journal of Clinical Virology , 27 (2), 136-145. https://doi.org/10.1016/S1386-6532(02)00168-3

Stenseth, N. C., Viljugrein, H., Jedrzejewski, W., Mysterud, A., \& Pucek, Z. (2002). Population dynamics of Clethrionomys glareolus and Apodemus flavicollis: seasonal components of density dependence and density independence. Acta Theriologica , 47 (S1), 39-67. https://doi.org/10.1007/BF03192479

Süss, J. (2011). Tick-borne encephalitis 2010: Epidemiology, risk areas, and virus strains in Europe and AsiaAn overview. Ticks and Tick-Borne Diseases , 2 (1), 2-15. https://doi.org/10.1016/j.ttbdis.2010.10.007

Tonteri, E., Jääskeläinen, A. E., Tikkakoski, T., Voutilainen, L., Niemimaa, J., Henttonen, H., ... Vapalahti, O. (2011). Tick-borne encephalitis virus in wild rodents in winter, Finland, 2008-2009. Emerging Infectious Diseases , 17 (1), 72-75. https://doi.org/10.3201/eid1701.100051

Velay, A., Argemi, X., Wendling, M.-J., Martinot, M., Hansmann, Y., \& Fafi-Kremer, S. (2019). L'encéphalite à tique en France : qu'en savons-nous aujourd'hui ? Revue Francophone des Laboratoires ,2019 (513), 34-43. https://doi.org/10.1016/S1773-035X(19)30287-4

Voordouw, M. J. (2015). Co-feeding transmission in Lyme disease pathogens. Parasitology , 142 (2), 290302. https://doi.org/10.1017/S0031182014001486

Woolhouse, M. E. J., Dye, C., Etard, J.-F., Smith, T., Charlwood, J. D., Garnett, G. P., .. Anderson, R. M. (1997). Heterogeneities in the transmission of infectious agents: Implications for the design of control programs. Proceedings of the National Academy of Sciences , 94 (1), 338-342. https://doi.org/10.1073/pnas.94.1.338

Zeipel, G. V., \& Heigl, Z. (1966). Experimental infection with tick-borne encephalitis virus in Clethrionomys glareolus, Apodemus flavicollis, Apodemus sylvaticus and Mus musculus. 2.Serological Studies. Acta Pathologica Microbiologica Scandinavica ,68 (2), 211-229. https://doi.org/10.1111/apm.1966.68.2.211

Zoldi, V., Papp, T., Rigo, K., Farkas, J., \& Egyed, L. (2015). A 4-year study of a natural tick-borne encephalitis virus focus in Hungary, 2010-2013. EcoHealth , 12 (1), 174-182. https://doi.org/10.1007/s10393014-0969-0 

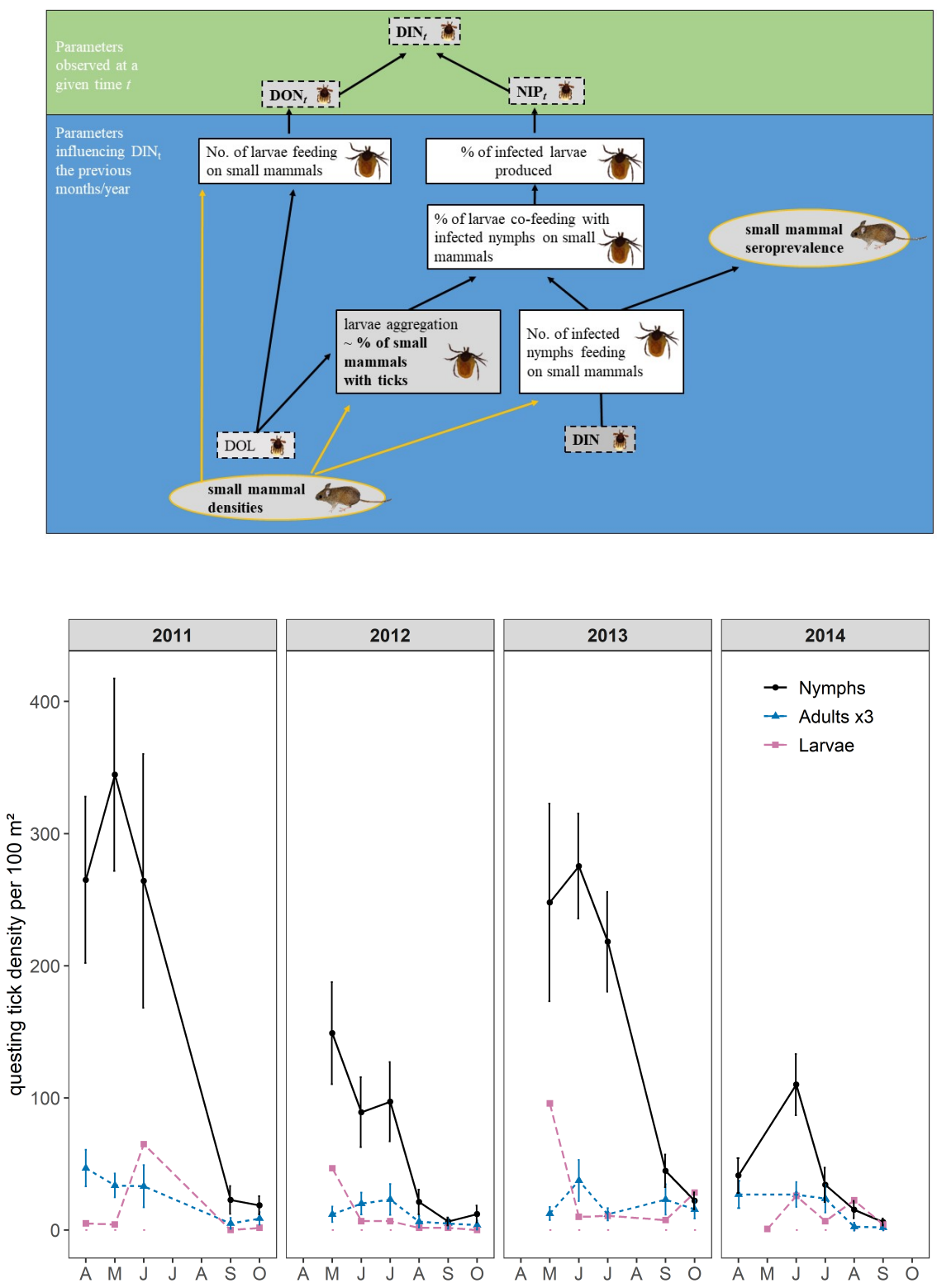

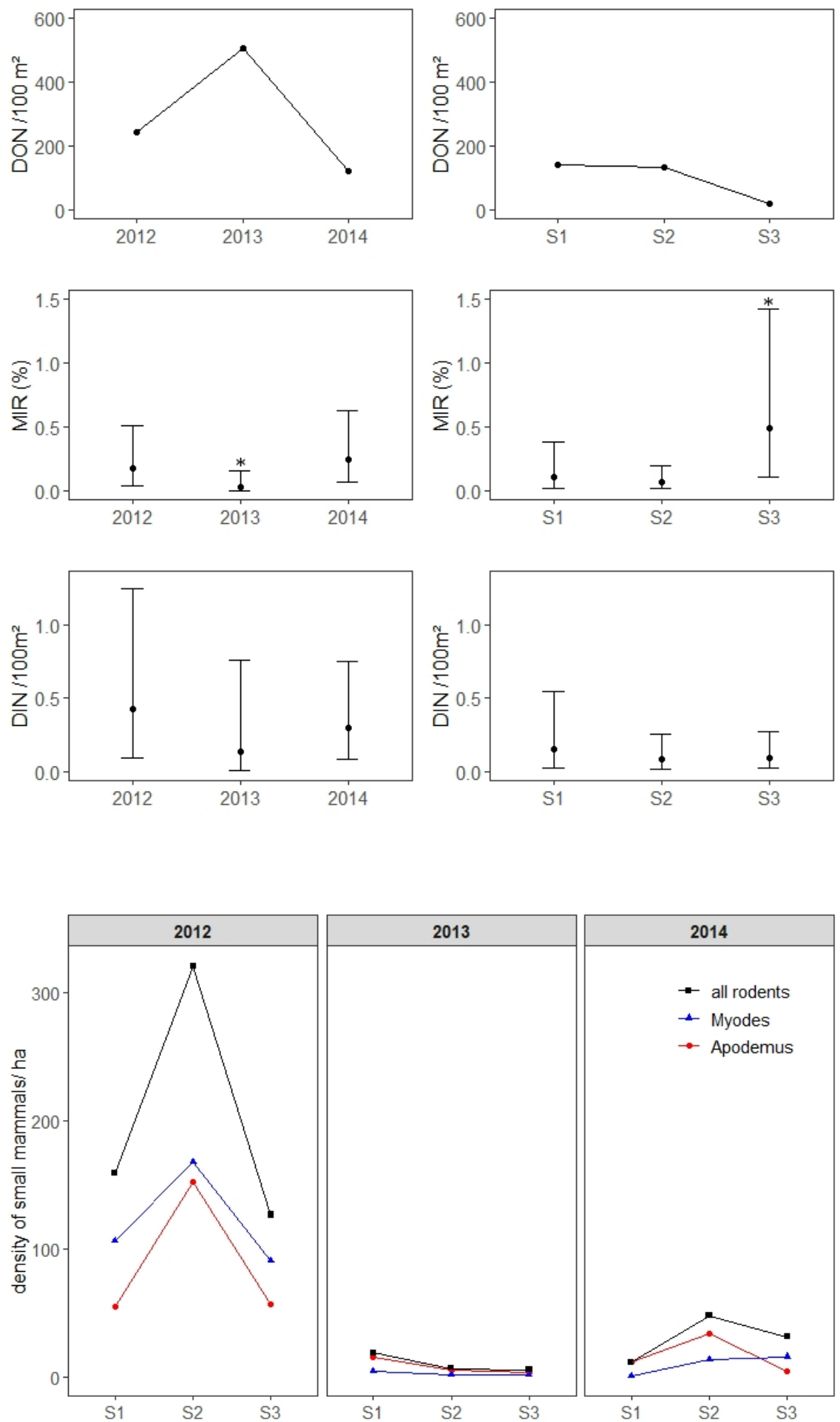

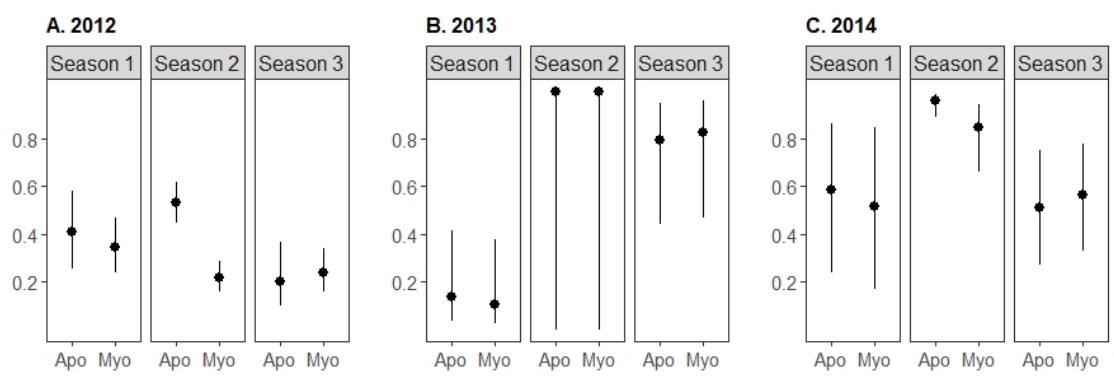

\section{Hosted file}

Bournez_Table1.xlsx available at https://authorea.com/users/309500/articles/440399-tick-borneencephalitis-virus-in-an-alsatian-forest-eastern-france-seasonal-and-annual-variation-ofepidemiological-parameters-related-to-nymph-to-larva-transmission-and-exposure-of-small-mammals

\section{Hosted file}

Bournez_Table2_Murbach.xlsx available at https://authorea.com/users/309500/articles/440399-tickborne-encephalitis-virus-in-an-alsatian-forest-eastern-france-seasonal-and-annual-variationof-epidemiological-parameters-related-to-nymph-to-larva-transmission-and-exposure-of-smallmammals 